\title{
Dietary and Household Sources of Prenatal Exposure to Polybrominated Diphenyl Ethers (PBDEs) in the INMA Birth Cohort (Spain)
}

Olga Costa, ${ }^{\dagger}$ Maria-Jose Lopez-Espinosa, ${ }^{*,+\dagger}$ Esther Vizcaino, ${ }^{\S}\|\|$ Mario Murcia, ${ }^{+, \dagger}$ Carmen Iñiguez, ${ }^{\dagger, \ddagger}$ Eva M. Navarrete-Muñoz, ${ }^{\ddagger}, \perp$ Joan O. Grimalt,, Adonina Tardon,, , Ferran Ballester, ${ }^{\dagger, \ddagger}$ and Ana Fernandez-Somoano ${ }^{\S, \ddagger}$

${ }^{\dagger}$ Epidemiology and Environmental Health Joint Research Unit, FISABIO-Universitat Jaume I-Universitat de València, Avenida de Catalunya 21, 46020 Valencia, Spain

"Spanish Consortium for Research on Epidemiology and Public Health (CIBERESP), Calle Monforte de Lemos 3-5, 28029 Madrid, Spain

${ }^{\S}$ Department of Preventive Medicine and Public Health, University of Oviedo, Campus del Cristo s/n, 33006 Oviedo, Asturias, Spain

"Department of Environmental Chemistry, Institute of Environmental Assessment and Water Research (IDÆA-CSIC), Jordi Girona 18, 08034 Barcelona, Spain

${ }^{\perp}$ Department of Public Health, Miguel Hernandez University, Ctra. Nacional 332, San Juan de Alicante, 03550 Alicante, Spain

Corresponding author: *Maria-Jose Lopez-Espinosa

Foundation for the Promotion of Health and Biomedical Research in the Valencian Region, FISABIO-Public Health, Avda Catalunya 21, 46020 Valencia, Spain. E-mail: lopez_josesp@gva.es; Phone: (+34) 961925941.

Number of pages: 8

Number of tables: 5 
Table S1. Definition of food groups: The Asturias and Valencia INMA Cohorts

(Spain), 2003-2008

\begin{tabular}{|c|c|}
\hline Food groups & Foods \\
\hline Eggs & Eggs \\
\hline Dairy products & $\begin{array}{l}\text { Whole milk; semi-skimmed milk; skimmed or low-fat milk; } \\
\text { condensed milk; full cream; whole fat yogurt; low-fat yogurt; } \\
\text { whole-fat cheese; low-fat cheese; custard, creme caramel, } \\
\text { pudding; and ice-cream }\end{array}$ \\
\hline Meat & $\begin{array}{l}\text { Chicken or turkey; game (duck, quail, and rabbit); beef, pork } \\
\text { or lamb; liver; offal; hamburger; ham, salami and others; } \\
\text { sausages; pate; and bacon }\end{array}$ \\
\hline Cereals and pasta & Breakfast cereals; corn; rice; and pasta \\
\hline Vegetables and pulses & $\begin{array}{l}\text { Spinach; cabbage, cauliflower or broccoli; lettuce or endive; } \\
\text { tomatoes; onions; carrots or squash; green beans; eggplant, } \\
\text { zucchini, or cucumber; green, red, or yellow peppers; } \\
\text { artichokes; asparagus; garlic; and pulses }\end{array}$ \\
\hline Fruits & $\begin{array}{l}\text { Oranges; orange juice; bananas; apples or pears; peaches, } \\
\text { nectarines, or apricots; watermelon or melon; grapes; prunes } \\
\text { or plums; kiwis; and olives }\end{array}$ \\
\hline Shellfish and cephalopods & $\begin{array}{l}\text { Crabs, shrimps, or lobster; clams or mussels; and squid and } \\
\text { octopus }\end{array}$ \\
\hline Fish & Lean fish; large oily fish; other oily fish; and other fish \\
\hline Lean fish & Hake, sole, gilthead and similar types of white fish \\
\hline Large oily fish & Swordfish, bonito, and fresh tuna; and canned tuna \\
\hline Other oily fish & $\begin{array}{l}\text { Mackerel, sardine, anchovy, or salmon; and canned sardine or } \\
\text { mackerel }\end{array}$ \\
\hline Other fish & $\begin{array}{l}\text { Assorted or mixed fried fish; dry or smoked fish; and surimi } \\
\text { and other fish-based food products }\end{array}$ \\
\hline
\end{tabular}


Table S2. Study population: The Asturias and Valencia INMA Cohorts (Spain), 2003-2008

\begin{tabular}{|c|c|c|c|c|}
\hline & Excluded & Included & $\mathbf{O R}^{\mathbf{a}}$ & $\mathbf{p}^{\mathbf{a}}$ \\
\hline & $(n=731)$ & $(n=541)$ & & \\
\hline Age: yrs & $30.3(4.6)$ & $30.8(4.5)$ & 0.99 & 0.61 \\
\hline Height: cm & $162.1(6.2)$ & $162.2(6.3)$ & 1.00 & 0.83 \\
\hline BMI: $\mathrm{kg} / \mathrm{m}^{2}$ & $23.9(4.7)$ & $23.7(4.3)$ & 0.99 & 0.45 \\
\hline Country of birth: $n(\%)$ & & & & 0.39 \\
\hline Spain & $654(90)$ & 507 (94) & ref & \\
\hline Other & $77(11)$ & $34(6)$ & 0.82 & \\
\hline Area of residence: $n(\%)$ & & & & 0.68 \\
\hline No rural & $674(92)$ & $489(90)$ & ref & \\
\hline Rural & $57(8)$ & $52(10)$ & 0.92 & \\
\hline Working ${ }^{\mathrm{b}}: n(\%)$ & & & & $<0.001$ \\
\hline No & $161(22)$ & 105 (19) & ref & \\
\hline Yes & $570(78)$ & $436(81)$ & 1.56 & \\
\hline Parity: $n(\%)$ & & & & 0.39 \\
\hline Primiparous & $405(55)$ & $324(60)$ & ref & \\
\hline Multiparous & $326(45)$ & $217(40)$ & 0.90 & \\
\hline Previous lactation: $n(\%)$ & & & & 0.87 \\
\hline None & $470(64)$ & $358(66)$ & ref & \\
\hline$<6$ months & $136(19)$ & 104 (19) & 1.00 & \\
\hline$\geq 6$ months & $125(17)$ & $78(14)$ & 1.09 & \\
\hline Education: $n(\%)$ & & & & 0.23 \\
\hline Until primary & $225(31)$ & 130 (24) & ref & \\
\hline Secondary & $324(44)$ & $227(42)$ & 0.96 & \\
\hline University & $182(25)$ & $184(34)$ & 1.22 & \\
\hline Social class ${ }^{\mathrm{c}}: n(\%)$ & & & & 0.31 \\
\hline I (highest) & $165(23)$ & $162(30)$ & ref & \\
\hline II (medium) & $195(27)$ & $127(24)$ & 0.81 & \\
\hline III (lowest) & $371(51)$ & $251(47)$ & 0.81 & \\
\hline Smoking $^{\mathrm{b}}$ & & & & 0.23 \\
\hline No & $429(60)$ & $358(68)$ & ref & \\
\hline Yes & $282(40)$ & $167(32)$ & 0.85 & \\
\hline Passive smoking ${ }^{\mathrm{d}}: n(\%)$ & & & & 0.33 \\
\hline No & $213(30)$ & $220(42)$ & ref & \\
\hline Yes & $492(70)$ & $301(58)$ & 0.88 & \\
\hline Alcohol intake ${ }^{\mathrm{b}}: n(\%)$ & & & & 0.93 \\
\hline No & $611(84)$ & 479 (89) & ref & \\
\hline Yes & $113(16)$ & $61(11)$ & 0.98 & \\
\hline
\end{tabular}

Values are $n(\%)$ or mean (standard deviation). BMI: Body mass index (before pregnancy). ${ }^{\mathrm{a}}$ Inclusion OR and p-value from logistic models adjusted by cohort; ${ }^{\mathrm{b}}$ At week 12 of pregnancy; ${ }^{\mathrm{c}}$ Class I: managerial jobs, senior technical staff, and commercial managers, Class II: skilled nonmanual workers, Class III: manual workers; ${ }^{\mathrm{d}}$ In at least two environments from among home, workplace, and leisure areas or restaurants. 
Table S3. Dietary and household-level factors: The Asturias and Valencia INMA Cohorts (Spain), 2003-2008

\begin{tabular}{|c|c|c|c|c|}
\hline & $\begin{array}{c}\text { Excluded } \\
(n=731)\end{array}$ & $\begin{array}{c}\text { Included } \\
(n=541)\end{array}$ & $\mathbf{O R}^{\mathbf{a}}$ & $\mathbf{p}^{\mathbf{a}}$ \\
\hline \multicolumn{5}{|l|}{ Dietary variables } \\
\hline Eggs: g/d & $20.1(8.2)$ & $20.8(8.2)$ & 1.01 & 0.18 \\
\hline Dairy products: g/d & $539.3(241.8)$ & $558.9(241.4)$ & 0.94 & 0.01 \\
\hline Meat: $\mathrm{g} / \mathrm{d}$ & $121.7(46.8)$ & $106.4(41.6)$ & 0.90 & 0.48 \\
\hline Cereals and pasta: $\mathrm{g} / \mathrm{d}$ & $111.1(43.8)$ & $95.3(43.1)$ & 0.78 & 0.09 \\
\hline Veg. and pulses: g/d & $246.9(117)$ & $245.6(121.2)$ & 0.96 & 0.40 \\
\hline Fruits: $\mathrm{g} / \mathrm{d}$ & $314.4(181.6)$ & $344.8(207.3)$ & 1.03 & 0.36 \\
\hline Shellfish and ceph.: g/d & $12.6(10.7)$ & $11.5(9.2)$ & 0.34 & 0.08 \\
\hline Fish: $\mathrm{g} / \mathrm{d}$ & $62.3(37)$ & $70.7(41.4)$ & 1.03 & 0.86 \\
\hline Lean fish: sv/wk & $1.2(1)$ & $1.2(1)$ & 1.02 & 0.73 \\
\hline Large oily fish: sv/wk & $2.2(1.5)$ & $2.4(1.6)$ & 0.97 & 0.50 \\
\hline Other oily fish: sv/wk & $0.6(0.9)$ & $0.6(0.8)$ & 0.95 & 0.53 \\
\hline Other fish: sv/wk & $1.3(1.4)$ & $1.5(1.3)$ & 0.98 & 0.71 \\
\hline \multicolumn{5}{|l|}{ Household variables } \\
\hline House size: $\mathrm{m}^{2}$ & $104.5(44.3)$ & $99.9(54.8)$ & 1.00 & 0.47 \\
\hline Curtains at home: $n(\%)$ & & & & 0.67 \\
\hline No & $33(5)$ & $33(6)$ & ref & \\
\hline Yes & $677(95)$ & $492(94)$ & 0.89 & \\
\hline Carpets at home: $n(\%)$ & & & & 0.48 \\
\hline No & $398(56)$ & $255(49)$ & ref & \\
\hline Yes & $309(44)$ & $270(51)$ & 0.91 & \\
\hline Mattress: $n(\%)$ & & & & 0.37 \\
\hline Innerspring & $455(67)$ & $322(64)$ & ref & \\
\hline Foam & $222(33)$ & $180(36)$ & 1.13 & \\
\hline Housekeeping: $n(\%)$ & & & & 0.46 \\
\hline$>1 \mathrm{t} / \mathrm{wk}$ & $391(55)$ & $326(62)$ & ref & \\
\hline$\leq 1 \mathrm{t} / \mathrm{wk}$ & $318(45)$ & $199(38)$ & 1.10 & \\
\hline TV use: h/wk & $16.2(8.9)$ & $15(7.9)$ & 1.00 & 0.88 \\
\hline \multicolumn{5}{|c|}{ Values are $n(\%)$ or mean (standard deviation). } \\
\hline \multicolumn{5}{|c|}{$\begin{array}{l}\text { Ceph.: cephalopods; g/d: grams per day; h/wk: hours per week; sv/w: } \\
\text { servings per week; t/wk: times per week; Veg.: Vegetables. }\end{array}$} \\
\hline \multicolumn{5}{|c|}{$\begin{array}{l}\text { atnclusion OR and p-value from logistic models adjusted by cohort. For } \\
\text { daily intake variables, OR are expressed for each } 100 \mathrm{~g} \text { increase in daily }\end{array}$} \\
\hline
\end{tabular}


Table S4. Sensitivity analysis: The Asturias and Valencia INMA Cohorts (Spain), 2003-2008

\begin{tabular}{|c|c|c|c|c|c|c|c|c|c|}
\hline & \multicolumn{3}{|c|}{ Main analysis $^{\mathrm{a}}$} & \multicolumn{3}{|c|}{ Adding lipids $^{\mathrm{a}}$} & \multicolumn{3}{|c|}{ Dichotomized BDE-209 } \\
\hline & \%change & $(95 \% \mathrm{CI})$ & $\mathbf{p}$ & \%change & $(95 \% \mathrm{CI})$ & $\mathbf{p}$ & OR & $(95 \% \mathrm{CI})$ & $\mathbf{p}$ \\
\hline \multicolumn{10}{|l|}{ BDE-47 } \\
\hline Shellfish and ceph. & 13.6 & $(0.03,29.0)$ & 0.050 & 9.6 & $(-3.3,24.2)$ & 0.152 & - & - & - \\
\hline Fish & 13.2 & $(-0.5,28.8)$ & 0.061 & 12.9 & $(-0.8,28.5)$ & 0.067 & - & - & - \\
\hline Large oily fish & 6.8 & $(-0.3,14.4)$ & 0.061 & 6.7 & $(-0.4,14.3)$ & 0.065 & - & - & - \\
\hline Foam mattress & 6.3 & $(-14.7,32.4)$ & 0.588 & 6.6 & $(-14.4,32.7)$ & 0.569 & - & - & - \\
\hline Housekeeping: > $1 \mathrm{t} / \mathrm{wk}$ & 15.7 & $(-6.9,43.8)$ & 0.188 & 15.8 & $(-6.8,43.8)$ & 0.186 & - & - & - \\
\hline \multicolumn{10}{|l|}{ BDE-99 } \\
\hline Shellfish and ceph. & 21.1 & $(2.3,43.5)$ & 0.027 & 18.6 & $(-0.0,40.6)$ & 0.051 & - & - & - \\
\hline Fish & 20.8 & $(1.7,43.4)$ & 0.032 & 19.7 & $(0.9,42.0)$ & 0.039 & - & - & - \\
\hline Large oily fish & 13.8 & $(4.0,24.7)$ & 0.005 & 13.6 & $(3.8,24.3)$ & 0.006 & - & - & - \\
\hline Foam mattress & -11.8 & $(-34.4,18.6)$ & 0.407 & -11.4 & $(-34.0,19.0)$ & 0.422 & - & - & - \\
\hline Housekeeping: $>1 \mathrm{t} / \mathrm{wk}$ & 2.9 & $(-22.8,37.1)$ & 0.847 & 3.2 & $(-22.4,37.4)$ & 0.827 & - & - & - \\
\hline \multicolumn{10}{|l|}{ BDE-153 } \\
\hline Shellfish and ceph. & 1.4 & $(-15.1,21.0)$ & 0.879 & 1.7 & $(-14.9,21.4)$ & 0.854 & - & - & - \\
\hline Fish & 8.2 & $(-9.4,29.2)$ & 0.387 & 8.4 & $(-9.2,29.5)$ & 0.372 & - & - & - \\
\hline Large oily fish & 8.8 & $(-1.2,19.7)$ & 0.086 & 8.8 & $(-1.1,19.8)$ & 0.083 & - & - & - \\
\hline Foam mattress & 8.2 & $(-19.6,45.7)$ & 0.602 & 8.2 & $(-19.6,45.6)$ & 0.605 & - & - & - \\
\hline Housekeeping: > $1 \mathrm{t} / \mathrm{wk}$ & 35.9 & $(0.4,83.9)$ & 0.046 & 35.9 & $(0.4,83.8)$ & 0.046 & - & - & - \\
\hline \multicolumn{10}{|l|}{ BDE-209 } \\
\hline Shellfish and ceph. & 21.7 & $(0.4,47.5)$ & 0.045 & 22.9 & $(1.3,49.0)$ & 0.036 & 1.023 & $(1.001,1.046)$ & 0.039 \\
\hline Fish & 14.9 & $(-7.1,42.2)$ & 0.200 & 15.2 & $(-6.9,42.6)$ & 0.192 & 1.003 & $(0.998,1.006)$ & 0.276 \\
\hline Large oily fish & 8.0 & $(-3.5,20.8)$ & 0.182 & 8.1 & $(-3.4,20.9)$ & 0.177 & 1.088 & $(0.942,1.253)$ & 0.248 \\
\hline Foam mattress & 48.9 & $(5.8,109.7)$ & 0.023 & 48.0 & $(5.2,108.3)$ & 0.025 & 1.593 & $(1.021,2.487)$ & 0.040 \\
\hline Housekeeping: > $1 \mathrm{t} / \mathrm{wk}$ & -14.7 & $(-39.5,20.2)$ & 0.364 & -14.8 & $(-39.5,20.1)$ & 0.363 & 0.903 & $(0.584,1.404)$ & 0.648 \\
\hline
\end{tabular}




\begin{tabular}{|c|c|c|c|c|c|c|c|c|c|}
\hline$\sum$ PBDEs & & & & & & & & & \\
\hline Shellfish and ceph. & 11.5 & $(2.2,21.7)$ & 0.015 & 11.5 & $(2.2,21.7)$ & 0.015 & - & - & - \\
\hline Fish & 9.1 & $(-0.03,19.0)$ & 0.051 & 9.0 & $(-0.1,19.0)$ & 0.053 & - & - & - \\
\hline Large oily fish & 5.7 & $(0.8,10.8)$ & 0.021 & 5.7 & $(0.8,10.8)$ & 0.022 & - & - & - \\
\hline Foam mattress & 8.9 & $(-6.0,26.2)$ & 0.255 & 9.0 & $(-5.9,26.2)$ & 0.253 & - & - & - \\
\hline Housekeeping: $>1 \mathrm{t} / \mathrm{wk}$ & 4.6 & $(-9.8,21.4)$ & 0.550 & 4.7 & $(-9.8,21.4)$ & 0.548 & _ & - & - \\
\hline
\end{tabular}

Ceph.: cephalopods; PBDE: Polybrominated diphenyl ether; t/wk: times per week; $\sum$ PBDEs: Sum of BDE-47, -99, -153, and -209.

${ }^{a}$ Percentage change and 95\% CI in cord serum PBDE concentrations per interquartile range (IQR) increment in daily intake of shellfish and cephalopods and fish (10.8 and $48.0 \mathrm{~g} /$ day, respectively) and per 1-serving increment in weekly intake of large oily fish. Percentage change and $95 \% \mathrm{CI}$ in cord serum PBDE concentrations with respect to the reference category, being "innerspring" for type of mattress and " $\leq 1$ times/week" for frequency of housekeeping. ${ }^{\mathrm{b}} \mathrm{OR}, 95 \% \mathrm{CI}$ and p-value from logistic models. Categories of dichotomized BDE-209: <LOD, $\geq$ LOD.

Models adjusted by alcohol intake (BDE-47, -99), working at week 12 of pregnancy (BDE-153, $\sum$ PBDEs), country of birth and season of the last menstrual period (BDE-209), and total energy intake (all models).

Results are only presented for those variables which were statistically significant in the main analysis. 
Table S5. Association between PBDEs and seafood intake or household-level factors stratified by cohort: The Asturias and Valencia INMA Cohorts (Spain), 2003-2008

\begin{tabular}{|c|c|c|c|c|c|c|}
\hline & \multicolumn{3}{|c|}{ Stratified: Asturias } & \multicolumn{3}{|c|}{ Stratified: Valencia } \\
\hline & \%change & $(95 \% \mathrm{CI})$ & $\mathbf{p}$ & \% change & $(95 \% \mathrm{CI})$ & $\mathbf{p}$ \\
\hline \multicolumn{7}{|l|}{ BDE-47 } \\
\hline Shellfish and ceph. & 13.9 & $(-8.3,41.5)$ & 0.241 & 11.4 & $(-5.6,31.4)$ & 0.203 \\
\hline Fish & 21.1 & $(-0.8,47.8)$ & 0.059 & -5.2 & $(-23.2,17.0)$ & 0.618 \\
\hline Large oily fish & 10.1 & $(-1.2,22.7)$ & 0.082 & 0.3 & $(-9.5,11.2)$ & 0.952 \\
\hline Foam mattress & 3.5 & $(-29.2,51.5)$ & 0.224 & 8.5 & $(-17.5,42.7)$ & 0.559 \\
\hline Housekeeping: > $1 \mathrm{t} / \mathrm{wk}$ & 29.2 & $(-14.5,95.5)$ & 0.223 & 8.7 & $(-15.4,39.6)$ & 0.514 \\
\hline \multicolumn{7}{|l|}{ BDE-99 } \\
\hline Shellfish and ceph. & 16.0 & $(-15.4,59.1)$ & 0.358 & 19.7 & $(-2.0,46.1)$ & 0.078 \\
\hline Fish & 19.3 & $(-10.4,58.9)$ & 0.226 & 8.4 & $(-16.1,40.0)$ & 0.537 \\
\hline Large oily fish & 15.6 & $(-0.9,34.9)$ & 0.066 & 8.5 & $(-4.1,22.9)$ & 0.197 \\
\hline Foam mattress & -29.9 & $(-60.4,24.2)$ & 0.221 & 3.6 & $(-25.6,44.2)$ & 0.834 \\
\hline Housekeeping: > $1 \mathrm{t} / \mathrm{wk}$ & -5.4 & $(-47.4,70.1)$ & 0.852 & 13.5 & $(-16.2,53.7)$ & 0.415 \\
\hline \multicolumn{7}{|l|}{ BDE-153 } \\
\hline Shellfish and ceph. & 3.8 & $(-18.0,31.4)$ & 0.757 & -10.8 & $(-33.1,19.0)$ & 0.437 \\
\hline Fish & 17.2 & $(-6.4,46.8)$ & 0.169 & -24.2 & $(-45.5,5.4)$ & 0.099 \\
\hline Large oily fish & 15.9 & $(2.4,31.1)$ & 0.020 & -8.6 & $(-22.3,7.4)$ & 0.272 \\
\hline Foam mattress & -11.8 & $(-42.8,35.9)$ & 0.568 & 36.7 & $(-8.8,105.0)$ & 0.130 \\
\hline Housekeeping: > $1 \mathrm{t} / \mathrm{wk}$ & 47.5 & $(-8.6,137.8)$ & 0.109 & 26.0 & $(-13.8,84.2)$ & 0.233 \\
\hline \multicolumn{7}{|l|}{ BDE-209 } \\
\hline Shellfish and ceph. & 19.4 & $(-18.8,75.7)$ & 0.367 & 23.5 & $(-1.0,54.1)$ & 0.062 \\
\hline Fish & 8.2 & $(-26.8,59.9)$ & 0.692 & 10.0 & $(-17.6,47.0)$ & 0.519 \\
\hline Large oily fish & 2.2 & $(-17.6,26.7)$ & 0.842 & 8.9 & $(-4.8,24.5)$ & 0.217 \\
\hline Foam mattress & 18.0 & $(-45.0,153.2)$ & 0.671 & 73.5 & $(23.2,144.4)$ & 0.002 \\
\hline Housekeeping: > $1 \mathrm{t} / \mathrm{wk}$ & -8.4 & $(-59.4,106.5)$ & 0.833 & -20.3 & $(-42.6,10.8)$ & 0.178 \\
\hline \multicolumn{7}{|l|}{$\sum$ PBDEs } \\
\hline Shellfish and ceph. & 8.1 & $(-2.9,20.3)$ & 0.157 & 13.1 & $(-3.1,31.9)$ & 0.119 \\
\hline Fish & 9.4 & $(-1.2,21.1)$ & 0.085 & -2.5 & $(-18.5,16.7)$ & 0.786 \\
\hline Large oily fish & 5.2 & $(-0.6,11.3)$ & 0.078 & 3.8 & $(-4.9,13.4)$ & 0.403 \\
\hline Foam mattress & 0.02 & $(-17.4,21.1)$ & 0.998 & 22.9 & $(-2.3,54.5)$ & 0.079 \\
\hline Housekeeping: >1 t/wk & 2.9 & $(-16.4,26.6)$ & 0.790 & 5.9 & $(-14.3,30.9)$ & 0.597 \\
\hline
\end{tabular}

Ceph.: cephalopods; PBDE: Polybrominated diphenyl ether; t/wk: times per week; $\sum$ PBDEs:

Sum of BDE-47, -99, -153, and -209. Percentage change and 95\% CI in cord serum PBDE concentrations per interquartile range (IQR) increment in daily intake of shellfish and cephalopods and fish (10.8 and $48.0 \mathrm{~g} /$ day, respectively) and per 1-serving increment in weekly intake of large oily fish. Percentage change and 95\% CI in cord serum PBDE concentrations with respect to the reference category, being "innerspring" for type of mattress and " $\leq 1$ times/week" for frequency of housekeeping. 
Models adjusted by alcohol intake (BDE-47, -99), working at week 12 of pregnancy (BDE-153, $\sum$ PBDEs), country of birth and season of the last menstrual period (BDE-209), and total energy intake (all models).

Results are only presented for those variables which were statistically significant in the joint models. 